\title{
Composição da entomofauna da Floresta Nacional do Araripe em diferentes vegetações e estações do ano
}

\author{
Francisco Roberto de Azevedo ${ }^{*}$, Maria Andréia Rodrigues de Moura ${ }^{2}$, Maria Solidade Barbosa Arrais ${ }^{3}$, \\ Daniel Rodrigues Nere ${ }^{2}$
}

\section{RESUMO}

A ocorrência de insetos tem grande significado ecológico e está relacionada com os fatores ambientais, disponibilidade de alimento e abrigo. Para avaliar a composição da entomofauna, em diferentes tipos de vegetação (Cerrado, Carrasco e Mata Úmida) e estações do ano na Floresta Nacional do Araripe, Crato, Ceará, nordeste brasileiro, foram realizadas coletas semanais na estação seca (setembro a dezembro) e chuvosa (abril a julho), por meio de armadilhas McPhail, de solo e bandejas amarelas. Os insetos da ordem Coleoptera são numerosos, na estação seca, agindo como polinizadores, fitófagos e detritívoros, além de decompositores de matéria orgânica, na estação chuvosa. Os Diptera são numerosos na estação chuvosa, quando são encontradas moscas frugívoras, decompositoras de carcaças de animais, de matéria orgânica e predadoras; os da família Calliphoridae predominam no Cerrado; da família Tachinidae, no Carrasco, e da Tephritidae, na Mata Úmida. Os Orthoptera Gryllidae predominam na Mata Úmida e os Hymenoptera Formicidae, no Carrasco e Cerrado na estação seca. Portanto, cada grupo de insetos desempenha um papel ecológico sobre as vegetações, nas diferentes estações do ano.

Palavras-chave: biodiversidade, indicadores ecológicos, ecossistemas, insetos florestais.

\section{ABSTRACT}

\section{Composition of entomofauna on the Araripe National Forest in different vegetation types and year seasons}

The occurrence of insects has great ecological significance and is related to environmental factors, food availability, and refuge. We assess the composition of the entomofauna in different vegetation types (cerrado, carrasco and humid forest), and seasons in the Araripe National Forest, Crato-CE, by weekly collections made in the dry season (September to December) and in the rainy season (April to July), through McPhail, pitfall and yellow tray traps. Many Coleoptera occur in the dry season, acting as pollinators, phytophagous, detritivore and decomposers of organic matter in the rainy season. Already, the Diptera is abundant in the rainy season, when fruit flies are found, decomposing animal carcasses, organic matter and predators. The Calliphoridae family predominate in the Cerrado, the Tachinidae in the Carrasco and Tephritidae in the Humid Forest. The Orthoptera Gryllidae predominate in the Humid Forest and the Hymenoptera Formicidae in the Carrasco and Cerrado in the dry season. Therefore, there is a satisfactory balance in the structure and functioning of the Araripe National Forest as each group plays an important ecological role on the vegetation, in the different seasons of the year.

Key words: Biodiversity, indicators ecological, ecosystems, forest insects.

Recebido para publicação em 11/03/2010 e aprovado em 10/11/2011

${ }^{1}$ Engenheiro-Agrônomo, Doutor. Universidade Federal do Ceará, Campus Cariri, Av. Tenente Raimundo Rocha, s/n Bairro Universitário, 63.040-360, Juazeiro do Norte, Ceará, Brasil.razevedo@ufc.br (*autor correspondente)

${ }^{2}$ Graduandos em Agronomia. Universidade Federal do Ceará, Campus Cariri, Av. Tenente Raimundo Rocha, s/n Bairro Universitário, 63.040-360, Juazeiro do Norte, Ceará, Brasil. andreiamoura.kd@bol.com.br; r.nere@agronomo.eng.br

${ }^{3}$ Graduanda em Ciências Biológicas. Universidade Regional do Cariri, Rua Cel. Antônio Luis, 1161, 63.100-000, Pimenta, Crato, Ceará, Brasil. soli-arrais@bol.com.br 


\section{INTRODUÇÃO}

Os insetos desempenham papel importante nos ecossistemas terrestres, pois estão envolvidos na decomposição de matéria orgânica, na ciclagem de nutrientes, no fluxo de energia, na polinização e na dispersão de sementes, além de serem reguladores de populações de plantas, de animais e de outros organismos (Lopes, 2008). O número de espécies de insetos em um ecossistema é o resultado de um equilíbrio que envolve muitos fatores, como as limitações ecológicas de natureza física, química ou biológica, sendo a vegetação determinante na biodiversidade (Ricklefs, 2001).

É importante reconhecer a entomofauna de certas áreas para que haja um constante acompanhamento dos impactos da ação antrópica nessas comunidades, já que o número de ordens, famílias e espécies de insetos diminui com a elevação do nível de antropização do ambiente (Thomanzini \& Thomanzini, 2002).

Nas florestas tropicais, a grande maioria das espécies de insetos e de outros animais é muito susceptível a processos de extinção, uma vez que ocorre em densidades populacionais muito baixas e participa de interações ecológicas às vezes muito estreitas e complexas com outras espécies. Muitos insetos são indicadores ecológicos da qualidade e da degradação ambiental, por causa das várias funções que desempenham na natureza, da estreita relação com a heterogeneidade dos ecossistemas e processos ecológicos, bem como por seu alto grau de sensibilidade às mudanças ambientais. Cada espécie responde de forma diferenciada a um distúrbio, sendo fundamental, portanto, reconhecer a sua interação com as alterações ambientais, bem como reconhecer e entender a sua evolução, tanto em locais degradados como em estágio de recuperação.

Os inventários e a identificação de insetos nos ecossistemas permitem as prevenções ou remediações de impactos nos diferentes ambientes. Isto caracteriza a importância de estudos que identifiquem grupos de indicadores ecológicos potenciais. Assim, à medida que ocorrem o resgate da diversidade vegetal e o equilíbrio ambiental, também os insetos respondem em diversidade e densidade, cumprindo a sua função indicadora (Wink et al., 2005).

Este trabalho teve como objetivo avaliar a composição da entomofauna presente nas vegetações de Mata Úmida, Cerrado e Carrasco da Floresta Nacional do Araripe, localizada no extremo sul do Estado do Ceará, na Chapada do Araripe, durante as estações seca e chuvosa.

\section{MATERIAL E MÉTODOS}

A pesquisa foi realizada na Floresta Nacional do Araripe (FLONA Araripe), em sua parte localizada na re- gião do Cariri cearense, município do Crato. Esta FLONA possui área de 39.262,326 hectares, apresentando clima tropical quente de seca, atenuado com estação chuvosa no outono, média pluviométrica de $1.000 \mathrm{~mm}$ anuais e temperatura oscilante entre 15 e $25^{\circ} \mathrm{C}$. A vegetação é subperenifólia de matas úmidas, com transição no sentido norte sul para o cerradão, cerrado e carrasco. O relevo é tabular, medindo cerca de 180 quilômetros de comprimento, no eixo leste/oeste, com uma variação de cerca 30 a 70 quilômetros de largura, no eixo norte/sul, com altitude mínima de 840 e máxima de 920 metros. Suas coordenadas geográficas são de $38^{\circ} 30^{\prime}$ a $40^{\circ} 55^{\prime}$ de longitude oeste de Greenwich e de $7^{\circ} 07^{\prime}$ a $7^{\circ} 49^{\prime}$ de latitude sul.

As coletas dos insetos foram realizadas, semanalmente, nos períodos de 18 de setembro a 4 de dezembro de 2008 (estação seca), e de 2 de abril a 7 de julho de 2009 (estação chuvosa), nas vegetações de Mata Úmida, Carrasco e Cerrado. A Mata Úmida é constituída por vegetação lenhosa de médio porte, com alguns elementos alcançando uma altura de 11 a 15 metros, fuste retilíneo, ramificações altas, apresentando um sub-bosque composto pela regeneração natural, muito densa. O Carrasco é formado por uma vegetação arbórea-arbustiva de pequeno porte, densa, apresentando um xeromorfismo acentuado com espécies caducifólias que alcançam uma altura de 5 metros. O Cerrado é formado por uma vegetação lenhosa mais esparsa de médio porte, com altura máxima de 11 metros, composta por elementos com fustes retilíneos e, ou tortuosos, bastante ramificados, sub-bosque com pequena incidência de regeneração natural (Lima et al., 1984). Nessas vegetações, foram empregados três métodos de coleta de insetos, descritos a seguir.

\section{Armadilhas McPhail}

Foram instaladas cinco armadilhas por cada tipo de vegetação, dispostas em duas fileiras de três e duas armadilhas, espaçadas de $30 \mathrm{~m}$ uma da outra $\left(3.600 \mathrm{~m}^{2}\right.$ de área amostrada), tendo, como atrativo alimentar, proteína hidrolisada de milho a 5\%. Em cada armadilha, foram usados $400 \mathrm{ml}$ da solução do atrativo e elas foram instaladas dentro da copa das árvores e, ou, arbustos, em local sombreado e a uma altura média de 1,8 $\mathrm{m}$ do solo.

\section{Armadilhas de solo}

Foram instaladas nove armadilhas de solo, em cada tipo de vegetação, dispostas em três linhas paralelas, contendo três armadilhas em cada linha, com espaçamentos de $25 \mathrm{~m}$ na linha e $30 \mathrm{~m}$ entre as linhas $\left(6.750 \mathrm{~m}^{2}\right.$ de área amostrada). Elas foram confeccionadas com garrafas plásticas de dois litros cortadas ao meio e com $10 \mathrm{~cm}$ de diâmetro e $15 \mathrm{~cm}$ de altura, mantendo-se sua abertura ao nível do solo. No interior de cada garrafa, foram colocados $250 \mathrm{ml}$ de uma solução de formol a 10\%, algumas gotas de 
detergente neutro e iscas de carne de frango em putrefação, penduradas acima da solução de formol, enroladas em um pedaço de organza e presas a um arame.

\section{Bandejas amarelas}

Foram instaladas seis bandejas em cada tipo de vegetação, dispostas em duas fileiras de três bandejas cada, espaçadas de $30 \mathrm{~m}$ uma da outra $\left(3.600 \mathrm{~m}^{2}\right.$ de área amostrada) e colocadas diretamente sobre o solo, contendo $500 \mathrm{ml}$ de uma solução de formol a $5 \%$ e algumas gotas de detergente neutro. Nas bordas das armadilhas, foram feitos orifícios onde foram colocadas telas finas para que, em caso de chuva, o excesso de água na bandeja não levasse os insetos coletados.

\section{Coleta dos insetos nas armadilhas}

As coletas dos insetos nas três armadilhas foram feitas semanalmente, substituindo-se a solução atrativa, as soluções de formol, assim como a carne em putrefação. Para recolher os insetos das armadilhas com solução líquida, utilizou-se uma peneira de plástico de malha fina. Realizaram-se quatro coletas mensais em cada vegetação, com cada tipo de armadilha e em cada estação do ano, totalizando 16 coletas/estação, sendo, portanto, coletado o ano inteiro. Os insetos coletados foram acondicionados em recipientes plásticos de $100 \mathrm{ml}$ contendo álcool $70 \%$ e, em seguida, levados ao laboratório de Entomologia da UFC, Campus Cariri, para triagem e identificação em nível de Ordem e Família, utilizando-se chaves ilustradas de identificação (Buzzi, 2008; Triplehorn \& Johnson, 2011).

\section{Análise faunística}

Cada vegetação foi considerada uma comunidade com características próprias, determinadas por meio dos seguintes índices faunísticos:

- Frequência: pi = ni/N, em que ni é o número de indivíduos da espécie i e N é o total de indivíduos da amostra (Silveira Neto et al., 1976).

- Dominância: $\mathrm{LD}=(1 / \mathrm{S})$ x 100, em que LD é o limite de dominância e $\mathrm{S}$ o número total de famílias. As famílias foram classificadas em dominantes, quando os valores da frequência apresentaram-se superiores a esse limite, e, não dominantes, quando os valores foram inferiores (Sakagami \& Laroca, 1971).

- Abundância: Para calcular a abundância, empregouse uma medida de dispersão, conforme Silveira Neto et al. (1976), por meio do cálculo do intervalo de confiança (IC) das médias das famílias, a $5 \%$ de probabilidade. Assim, as famílias foram classificadas em raras, quando o número de indivíduos foi menor que o limite inferior do IC; dispersas, quando esse número ficou entre os limites inferiores do IC; comuns, quando situado dentro do IC; abundantes, quando entre os limites superiores; e muito abundan- tes, quando o número de indivíduos foi maior que o limite superior do IC.

- Constância: C = p x 100/N, em que p é o número de coletas com a família e $\mathrm{N}$ é o número total de amostras tomadas (Silveira Neto et al., 1976). As famílias são classificadas como constantes, quando estão presentes em mais de $50 \%$ das amostras; acessórias, quando presentes em 25-50\% das amostras; e acidentais, quando presentes em menos de $25 \%$ das amostras.

- Riqueza: Refere-se à abundância numérica de uma determinada família em uma comunidade e pode ser calculada pelo índice de Menhinick (Whittaker, 1977): $\mathrm{D}_{\mathrm{Mn}}=\mathrm{S} / \mathrm{VN}$, em que $\mathrm{S}$ é o número de famílias e $\mathrm{N}$ o de indivíduos na comunidade.

\section{RESULTADOS E DISCUSSÃO}

\section{Insetos coletados na estação seca}

$\mathrm{Na}$ estação seca, foram amostradas um total de 22 famílias, pertencentes a sete ordens. A ordem Coleoptera foi a mais rica, com sete famílias, seguida da ordem Diptera, com quatro (Tabela 1). Dos 5.218 indivíduos capturados nessa estação, 35,61\% foram encontrados na Mata Úmida, 33, $17 \%$ no Carrasco e 31,22\% no Cerrado.

Os besouros possuem grande importância ecológica, auxiliando na percepção das condições ambientais locais de uma fitofisionomia (Chung et al., 2000) e a sua diversidade está relacionada com a composição e a estrutura da vegetação, revelando um mecanismo natural de atração, abrigo e alimentação (Schorn, 2000). Estes insetos interagem nos ecossistemas florestais por meio de associações com frutos e, ou, sementes de espécies florestais arbóreas (Zidko, 2002).

Os Hymenoptera, Hemiptera e Orthoptera apresentaram a mesma riqueza (três famílias), enquanto as ordens Blattodea e Dermaptera foram às menos ricas, com apenas uma família cada.

Na Mata Úmida, os ortópteros da família Gryllidae foram os mais frequentes $(23,47 \%)$, seguidos dos Hymenoptera Formicidae (16\%), Coleoptera Curculionidae (14\%) e Diptera Tachinidae (14\%). Todas essas famílias foram dominantes e muito abundantes. No entanto, as duas primeiras foram constantes, enquanto as duas últimas acessórias (Tabela 2).

Essa vegetação, por apresentar um ambiente fechado e com temperaturas amenas, certamente propiciou condições ideais para os grilos, graças a maior concentração de serrapilheira e de matéria orgânica. A fauna desses insetos é pobre em espécies e sua abundância é relativamente baixa em fragmentos de florestas (Sperber, 1999). Perturbações físicas causam respostas rápidas na comunidade de grilos, podendo ser indicadores em uma escala local (Sperber et al., 2007). 
A estrutura das comunidades das formigas é fundamental em estudo de impacto ambiental, pois estas mantêm e restauram a qualidade do solo. Elas operam na redistribuição das partículas, dos nutrientes e da matéria orgânica, além de melhorar a infiltração de água no solo pelo aumento da porosidade e a aeração (Bruyn, 1999). São dominantes na maioria dos ecossistemas, estando presentes nos mais diferentes habitats. Sua riqueza de espécies está correlacionada com o tipo e a variedade da vegetação, sendo que o aumento na complexidade da vegetação garante aumento na sua diversidade (Soares $e t$ $a l .$, 2001), condições essas encontradas na Mata Úmida.

Os besouros curculionídeos habitam toda a terra onde exista vegetação terrestre e representam a maior família do reino animal, com aproximadamente 62.000 espécies descritas. Seu sucesso evolutivo está no fato de serem endofitófagos e suas larvas alimentam-se de uma grande variedade de estruturas das plantas (Oberprieler et $a l ., 2007)$. Portanto, as espécies que vivem na Mata Úmida podem estar associadas tanto às estruturas vegetativas, como às estruturas reprodutivas das plantas dessa vegetação.

A maioria dos taquinídeos são parasitóides de lagartas de Lepidoptera ou larvas de Coleoptera, porém alguns atacam Hemiptera, Orthoptera e algumas outras ordens (Triplehorn \& Jonnson, 2011). Como nessa vegetação houve maior freqüência de insetos fitófagos como os grilos e os bicudos, acredita-se que essas moscas desempenharam um papel importante nessa vegetação como regulador das populações dos insetos.

Os Diptera das famílias Drosophilidae e Muscidae apresentaram frequências de 8 e $6 \%$, respectivamente. Foram muito abundantes, mas não dominaram nessa vegetação. Apesar disso, esses insetos têm sua função biológica na decomposição da matéria orgânica da Mata Úmida, porque atuam sobre frutos fermentados e sobre carcaças de animais silvestres, respectivamente.

As baratas da família Blattidae, apesar de constantes, demonstraram baixa frequência, não dominância, e foram dispersas na vegetação. As chamadas baratas de madeira também são consideradas importantes na decomposição de árvores mortas caídas na mata. As demais famílias de insetos capturados na estação seca e na Mata Úmida foram menos frequentes, não dominantes, dispersas e acessórias na vegetação e algumas estiveram ausentes (Tabela 2).

No Carrasco, os insetos que mais se destacaram foram os Formicidae, com 21,37\% de frequência, seguido dos Drosophilidae (14,38\%) e Curculionidae (10,57\%), sendo

Tabela 1. Número total de indivíduos, por ordem e família, capturados na estação seca em três vegetações da Floresta Nacional do Araripe, Crato, Ceará

\begin{tabular}{|c|c|c|c|c|c|}
\hline \multirow{2}{*}{ Ordem } & \multirow{2}{*}{ Família } & \multicolumn{4}{|c|}{ Vegetações } \\
\hline & & Mata Úmida & Carrasco & Cerrado & Total \\
\hline \multirow{4}{*}{ Diptera } & Drosophilidae & 144 & 249 & 317 & 710 \\
\hline & Muscidae & 108 & 123 & 271 & 502 \\
\hline & Tachinidae & 254 & 144 & 25 & 423 \\
\hline & Lonchaeidae & 0 & 64 & 0 & 64 \\
\hline \multirow{7}{*}{ Coleoptera } & Curculionidae & 258 & 183 & 14 & 455 \\
\hline & Nitidulidae & 74 & 18 & 117 & 209 \\
\hline & Scarabaeidae & 14 & 92 & 7 & 113 \\
\hline & Chrysomelidae & 10 & 30 & 4 & 44 \\
\hline & Staphylinidae & 7 & 9 & 7 & 23 \\
\hline & Erotylidae & 0 & 17 & 0 & 17 \\
\hline & Melyridae & 14 & 0 & 0 & 14 \\
\hline \multirow{3}{*}{ Hymenoptera } & Formicidae & 294 & 370 & 324 & 988 \\
\hline & Vespidae & 38 & 22 & 3 & 63 \\
\hline & Ichneumonidae & 10 & 19 & 21 & 50 \\
\hline Blattodea & Blattidae & 64 & 123 & 128 & 315 \\
\hline \multirow{3}{*}{ Hemiptera } & Cicadellidae & 0 & 81 & 269 & 350 \\
\hline & Cydnidae & 45 & 62 & 11 & 118 \\
\hline & Pentatomidae & 23 & 0 & 4 & 27 \\
\hline \multirow{3}{*}{ Orthoptera } & Gryllidae & 436 & 87 & 74 & 597 \\
\hline & Stenopelmatidae & 12 & 18 & 7 & 37 \\
\hline & Acrididae & 5 & 7 & 13 & 25 \\
\hline Dermaptera & Forficulidae & 24 & 13 & 0 & 37 \\
\hline Total & & 1.858 & 1.731 & 1.629 & 5.218 \\
\hline
\end{tabular}


que apenas as duas primeiras famílias foram dominantes e constantes, mas todas muito abundantes (Tabela 2). Como essa vegetação é uma transição da Mata Úmida, observase a ocorrência de certas famílias com frequências semelhantes às da vegetação anterior, como os Formicidae (16\%) e Curculionidae (14\%).

Os Gryllidae e as cigarrinhas da família Cicadellidae foram comuns e acessórias na vegetação do Carrasco, porém não dominaram e foram pouco frequentes. Nessa vegetação, por haver menor concentração de serrapilheira, desfavoreceu-se a ocorrência dos grilos. Os Muscidae, Tachinidae, Blattidae e Scarabaeidae, apesar de muito abundantes, também não dominaram, sendo as duas primeiras famílias de ocorrência acessória, a terceira constante e a última acidental (Tabela 2).

Os besouros escarabeídeos são importantes em estudos de fragmentos vegetais, pois se alimentam de fezes e carcaças dos vertebrados (Thomanzini \& Thomanzini, 2000). Por terem natureza sedentária, esses coleópteros são mais vulneráveis a mudanças ambientais (Kimberling et al., 2001) e a sua distribuição local é fortemente influenciada pela cobertura vegetal (Halter \& Arellano, 2002); deste modo, esse grupo é indicador ecológico importante por responder às diferenças estruturais entre fitofisiono- mias diferentes. Eles também são detritívoros, promovendo a remoção e reingresso da matéria orgânica no ciclo de nutrientes, aumentando a aeração do solo e prolongando a sua capacidade produtiva (Milhomem et al., 2003).

As demais famílias apresentaram baixíssimas frequências e foram não dominantes, dispersas e de ocorrência acidental, na vegetação, e algumas estiveram ausentes (Tabela 2).

No Cerrado, as famílias Formicidae e Drosophilidae apresentaram frequências semelhantes, com 19,89 e $19,45 \%$, respectivamente, seguidas de Muscidae $(16,64 \%)$ e Cicadellidae (16,51\%). Destas, todas foram dominantes, muito abundantes, mas somente as formigas foram constantes; as outras famílias encontradas foram acessórias (Tabela 2).

Alguns padrões de variação espacial e temporal já foram descritos para as comunidades de drosofilídeos do Cerrado brasileiro (Tidon, 2006), mas os mecanismos responsáveis por esses padrões ainda são pouco conhecidos. Essas moscas são conhecidas por se alimentarem em uma ampla variedade de substratos orgânicos, incluindo frutos em decomposição, flores e fungos, entre outros (Shorrocks, 1974). Como no Cerrado Caririense existe uma grande diversidade de frutos silvestres, como o pequi

Tabela 2. Análise faunística das famílias de insetos capturados na estação seca em três vegetações da Floresta Nacional do Araripe, Crato, Ceará

\begin{tabular}{|c|c|c|c|c|c|c|c|c|c|c|c|c|}
\hline \multirow{3}{*}{ Famílias } & \multicolumn{12}{|c|}{ Vegetações } \\
\hline & \multicolumn{4}{|c|}{ Mata Úmida } & \multicolumn{4}{|c|}{ Carrasco } & \multicolumn{4}{|c|}{ Cerrado } \\
\hline & $\mathbf{F}(\%)$ & D & $\mathbf{A}$ & $\mathrm{C}(\%)$ & $\mathbf{F}(\%)$ & D & $\mathbf{A}$ & $\mathrm{C}(\%)$ & $\mathrm{F}(\%)$ & D & $\mathbf{A}$ & $\mathrm{C}(\%)$ \\
\hline Formicidae & 16,00 & $\mathrm{D}$ & $\mathrm{ma}$ & $66,67 \mathrm{c}$ & 21,37 & $\mathrm{D}$ & $\mathrm{ma}$ & $66,66 c$ & 19,89 & $\mathrm{D}$ & $\mathrm{ma}$ & $66,67 \mathrm{c}$ \\
\hline Drosophilidae & 8,00 & nd & $\mathrm{ma}$ & $8,33 \mathrm{ac}$ & 14,38 & $\mathrm{D}$ & $\mathrm{ma}$ & $55 c$ & 19,45 & $\mathrm{D}$ & $\mathrm{ma}$ & $26,67 a$ \\
\hline Gryllidae & 23,47 & $\mathrm{D}$ & $\mathrm{ma}$ & $75 c$ & 5,03 & nd & $\mathrm{c}$ & $41,67 \mathrm{a}$ & 4,54 & nd & $\mathrm{d}$ & $52 \mathrm{a}$ \\
\hline Muscidae & 6,00 & nd & $\mathrm{ma}$ & $7,54 \mathrm{ac}$ & 7,11 & nd & $\mathrm{ma}$ & $8,33 \mathrm{ac}$ & 16,64 & $\mathrm{D}$ & $\mathrm{ma}$ & $57,33 a$ \\
\hline Curculionidae & 14,00 & $\mathrm{D}$ & $\mathrm{ma}$ & $33,33 a$ & 10,57 & nd & $\mathrm{ma}$ & $25 \mathrm{a}$ & 0,86 & o & $\mathrm{o}$ & o \\
\hline Tachinidae & 14,00 & $\mathrm{D}$ & $\mathrm{ma}$ & $25 \mathrm{a}$ & 8,32 & nd & $\mathrm{ma}$ & $41,67 \mathrm{a}$ & 1,53 & nd & d & $55 \mathrm{c}$ \\
\hline Cicadellidae & o & o & o & o & 4,68 & nd & $\mathrm{c}$ & $33,33 a$ & 16,51 & $\mathrm{D}$ & $\mathrm{ma}$ & $33,33 a$ \\
\hline Blattidae & 3,44 & nd & $\mathrm{d}$ & $66,67 \mathrm{c}$ & 7,11 & nd & $\mathrm{ma}$ & $66,67 \mathrm{c}$ & 8,00 & nd & $\mathrm{ma}$ & $66,67 \mathrm{c}$ \\
\hline Nitidulidae & 4,00 & nd & $\mathrm{d}$ & $8,33 \mathrm{ac}$ & 1,04 & nd & $\mathrm{d}$ & $8,33 \mathrm{ac}$ & 7,18 & nd & $\mathrm{ma}$ & $66,67 \mathrm{c}$ \\
\hline Cydnidae & 2,42 & nd & $\mathrm{d}$ & $5,33 \mathrm{ac}$ & 3,58 & nd & $\mathrm{d}$ & $8,33 \mathrm{ac}$ & 0,67 & nd & $\mathrm{d}$ & $25 \mathrm{ac}$ \\
\hline Scarabaeidae & 1,00 & $\mathrm{o}$ & o & o & 5,31 & nd & $\mathrm{ma}$ & $8,33 a c$ & 0,43 & o & o & $\mathrm{o}$ \\
\hline Lonchaeidae & o & o & o & o & $\mathrm{o}$ & 3,70 & nd & $\mathrm{d}$ & $7,5 \mathrm{ac}$ & 0,10 & o & o \\
\hline Vespidae & 2,05 & nd & $\mathrm{d}$ & $8,33 \mathrm{ac}$ & 1,27 & nd & $\mathrm{d}$ & $5,5 \mathrm{ac}$ & 0,18 & o & o & o \\
\hline Ichneumonidae & 0,54 & o & o & o & 1,10 & nd & $\mathrm{d}$ & $50 \mathrm{c}$ & 1,30 & nd & d & $8,33 \mathrm{ac}$ \\
\hline Chrysomelidae & 0,54 & o & o & o & 1,73 & nd & $\mathrm{d}$ & $\mathrm{ac}$ & 0,25 & o & o & o \\
\hline Forficulidae & 1,29 & nd & $\mathrm{d}$ & $8,33 \mathrm{ac}$ & 0,75 & o & $\mathrm{O}$ & $\mathrm{o}$ & 0,45 & o & o & o \\
\hline Stenopelmatidae & 0,65 & o & o & o & 1,04 & nd & $\mathrm{d}$ & $\mathrm{ac}$ & 0,43 & o & o & o \\
\hline Pentatomidae & 1,24 & nd & $\mathrm{d}$ & $8,33 \mathrm{ac}$ & o & o & o & o & 0,25 & o & o & o \\
\hline Acrididae & 0,27 & o & o & o & o & o & o & o & 1,00 & nd & $\mathrm{d}$ & $8,33 \mathrm{ac}$ \\
\hline Staphylinidae & 0,38 & o & o & $\mathrm{o}$ & 1,0 & nd & $\mathrm{d}$ & $8,33 \mathrm{ac}$ & 0,43 & $\mathrm{o}$ & o & o \\
\hline Erotylidae & o & o & o & o & 0,98 & nd & $\mathrm{d}$ & $7,5 \mathrm{ac}$ & $\mathrm{o}$ & o & o & o \\
\hline Melyridae & 0,75 & nd & $\mathrm{d}$ & $8,33 \mathrm{ac}$ & o & o & $\mathrm{o}$ & o & o & o & o & o \\
\hline
\end{tabular}

$\mathrm{F}=$ Frequência $; \mathrm{D}=$ Dominância: $\mathrm{D}=$ dominante; $\mathrm{nd}=$ não dominante; $\mathrm{A}=$ Abundância: $\mathrm{c}=$ comum; $\mathrm{d}=$ dispersa; ma $=$ muito abundante; $\mathrm{C}=$ Constância: $\mathrm{c}=$ constante; $\mathrm{a}=$ acessória; $\mathrm{ac}=$ acidental; $\mathrm{o}=$ sem ocorrência. 
(Caryocar brasiliense Cambess.), a seriguela (Spondias purpurea L.), o cajá (Spondias lutea L.), o araçá (Psidium araca Raddi), a mangaba (Hancornia speciosa Gomes), o juazeiro (Ziziphus joazeiro Mart.), a carnaúba (Copernicia prunifera (Mill.) H.E. Moore), o maracujá (Passiflora edulis Sims) e outras, isso faz com que esses insetos tenham grande importância na ciclagem de nutrientes, nessa vegetação, na estação seca.

Por seu hábito fitossuccívoro, as cigarrinhas representam um grupo com grande potencial para estudos relacionados à biodiversidade florestal e como indicadoras das alterações na composição vegetal (Coelho \& Silva, 2003). Esses insetos preferem habitat constituído por vegetações baixas (Vaz et al., 2009) e, provavelmente, pelo fato da vegetação do Cerrado apresentar plantas mais baixas, essa condição propiciou maior atividade de voo dessas cigarrinhas entre as plantas e maior captura nas bandejas, quando comparado com a do Carrasco e a da Mata Úmida.

Os Blattidae e os coleópteros Nitidulidae mantiveramse com frequências acima de 7\%, foram constantes e muito abundantes na vegetação de Cerrado, porém não dominaram. Os nitidulídeos são os principais polinizadores das anonáceas cultivadas, como a pinha e graviola (Nadel \& Peña, 1994), e a maioria dos autores descreve essa família como uma das mais frequentes na polinização de palmeiras (Küchmeister et al., 1998). Além de anonáceas silvestres, ocorrem também palmeiras na FLONA Araripe. A dispersão e a polinização são processos ecológicos estratégicos em comunidades florestais; seu estudo tem grande importância no entendimento das variáveis envolvidas na organização da comunidade (Yamamoto, 2001). Portanto, esses insetos têm um papel importante na polinização das plantas da vegetação do Cerrado caririense.

As moscas da família Tachinidae ocorreram de forma acessória nessa vegetação, enquanto os percevejos Cydnidae, os himenópteros Ichneumonidae e os gafanhotos Acrididae, de forma acidental, apresentando-se todos com baixa frequência, não dominância e dispersão nessa vegetação. As demais famílias estiveram ausentes (Tabela 2).

\section{Insetos coletados na estação chuvosa}

Na estação chuvosa, foram amostradas um total de 21 famílias, pertencentes, também, a sete ordens. Nessa estação, a ordem Diptera foi a mais rica, com seis famílias, seguida das Coleoptera e Hymenoptera, com quatro famílias cada. As Hemiptera e Orthoptera apresentaram a mesma riqueza (três famílias), enquanto as Blattodea e Dermaptera foram as menos ricas, com apenas uma família

Tabela 3. Número total de indivíduos, por ordem e família, capturados na estação chuvosa em três vegetações da Floresta Nacional do Araripe, Crato, Ceará

\begin{tabular}{|c|c|c|c|c|c|}
\hline \multirow{2}{*}{ Ordem } & \multirow{2}{*}{ Família } & \multicolumn{4}{|c|}{ Vegetações } \\
\hline & & Mata Úmida & Carrasco & Cerrado & Total \\
\hline \multirow{6}{*}{ Diptera } & Calliphoridae & 808 & 2.138 & 10.910 & 13.856 \\
\hline & Tachinidae & 0 & 5.463 & 4.292 & 9.755 \\
\hline & Sarcophagidae & 277 & 982 & 2.067 & 3.326 \\
\hline & Tephritidae & 2.182 & 46 & 4 & 2.232 \\
\hline & Stratiomyidae & 0 & 0 & 1.605 & 1.605 \\
\hline & Drosophilidae & 0 & 109 & 36 & 145 \\
\hline \multirow{4}{*}{ Coleoptera } & Scolytidae & 784 & 0 & 0 & 784 \\
\hline & Chrysomelidae & 1 & 0 & 110 & 111 \\
\hline & Curculionidae & 8 & 8 & 63 & 79 \\
\hline & Carabidae & 0 & 0 & 28 & 28 \\
\hline \multirow{4}{*}{ Hymenoptera } & Formicidae & 407 & 321 & 188 & 916 \\
\hline & Ichneumonidae & 127 & 76 & 2 & 205 \\
\hline & Sphecidae & 0 & 0 & 49 & 49 \\
\hline & Scoliidae & 0 & 0 & 47 & 47 \\
\hline Blattodea & Blattidae & 451 & 172 & 297 & 920 \\
\hline \multirow{3}{*}{ Hemiptera } & Cicadellidae & 87 & 204 & 116 & 407 \\
\hline & Reduvidae & 1 & 6 & 7 & 14 \\
\hline & Cercopidae & 0 & 0 & 11 & 11 \\
\hline \multirow{2}{*}{ Orthoptera } & Gryllidae & 389 & 46 & 227 & 662 \\
\hline & Stenopelmatidae & 0 & 0 & 62 & 62 \\
\hline \multirow[t]{2}{*}{ Dermaptera } & Forficulidae & 44 & 10 & 1 & 55 \\
\hline & & 5.610 & 9.591 & 20.122 & 35.323 \\
\hline
\end{tabular}


cada (Tabela 3). Dos 35.323 indivíduos capturados nessa estação, 56,97\% foram encontrados no Cerrado, 27,15\% no Carrasco e $15,88 \%$ na Mata Úmida.

No Cerrado, a família mais frequente foi Calliphoridae $(54,22 \%)$, constante na vegetação, seguida de Tachinidae $(21,33 \%)$ e Sarcophagidae $(10,27 \%)$, de ocorrências acessórias. Elas também foram dominantes e muito abundantes nessa vegetação, assim como, os Stratiomyidae, mas de ocorrência acidental (Tabela 4).

Os insetos necrófagos, como as moscas califorídeas e sarcofagídeas, são os primeiros a chegarem às carcaças dos animais para a realização de oviposições e alimentação dos adultos, minutos após a morte (Smith, 1986), permanecendo na carcaça ao longo dos estádios de decomposição (Catts \& Goff, 1992). Como no Cerrado existe uma grande diversidade de animais silvestres mortos, é de suma importância a presença dessas famílias para a ciclagem de energia na vegetação.

Os dípteros Tachinidae, por serem parasitoides, desempenham importante papel no equilíbrio de populações de insetos fitófagos nessa vegetação. Paschoalini et al. (2004), ao realizarem estudo no Parque Estadual do Rio Doce, Estado de Minas Gerais, utilizando armadilha malaise, em condições do cerrado, coletaram um total de 25.298 indivíduos, pertencentes a 27 famílias, sendo que uma das mais frequentes foram Tachinidae, com 11,68\% do total.

As larvas de moscas Stratiomyidae estão associadas à decomposição de matéria orgânica vegetal (Pujol-Luz et al., 2004). Rozkosny (1982) verificou o mesmo em adultos de espécies europeias, geralmente encontradas em vegetação rasteira, como no Cerrado. Nesta família, encontramse larvas coprófagas e sarconecrofitófagas, com participação no processo de reciclagem da matéria orgânica vegetal. Daí sua importância no Cerrado e na estação chuvosa, juntamente com os decompositores de matéria orgânica animal.

As famílias Blattidae, Formicidae e Gryllidae foram constantes, mas não dominaram e foram dispersas na vegetação. Já as famílias Cicadellidae, Drosophilidae, Chrysomelidae, Curculionidae e Stenopelmatidae ocorreram de forma acessória nessa vegetação, enquanto as famílias Sphecidae, Scoliidae, Carabidae, Reduvidae e Cercopidae ocorreram de forma acidental e todas, também, não dominaram e foram dispersas na vegetação.

No Carrasco, a família mais frequente foi Tachinidae (57\%), seguida de Calliphoridae (22,30\%) e Sarcophagidae $(10,54 \%)$. Elas também foram dominantes, muito abundantes e constantes na vegetação do carrasco (Tabela 4). Observou-se nessa vegetação grande surto de carrapa-

Tabela 4. Análise faunística das famílias de insetos capturados na estação chuvosa em três vegetações da Floresta Nacional do Araripe, Crato, Ceará

\begin{tabular}{|c|c|c|c|c|c|c|c|c|c|c|c|c|}
\hline \multirow{3}{*}{ Famílias } & \multicolumn{12}{|c|}{ Vegetações } \\
\hline & \multicolumn{4}{|c|}{ Mata Úmida } & \multicolumn{4}{|c|}{ Carrasco } & \multicolumn{4}{|c|}{ Cerrado } \\
\hline & $\mathbf{F}(\%)$ & D & $\mathbf{A}$ & $\mathrm{C}(\%)$ & $\mathbf{F}(\%)$ & D & $\mathbf{A}$ & $\mathrm{C}(\%)$ & $\mathbf{F}(\%)$ & D & $\mathbf{A}$ & $\mathrm{C}(\%)$ \\
\hline Calliphoridae & 14,40 & $\mathrm{D}$ & $\mathrm{ma}$ & $58,33 \mathrm{c}$ & 22,30 & $\mathrm{D}$ & $\mathrm{ma}$ & $58,33 \mathrm{c}$ & 54,22 & $\mathrm{D}$ & $\mathrm{ma}$ & $58,33 \mathrm{c}$ \\
\hline Tachinidae & o & o & o & o & 57,00 & $\mathrm{D}$ & $\mathrm{ma}$ & $51 \mathrm{c}$ & 21,33 & $\mathrm{D}$ & ma & $25 \mathrm{a}$ \\
\hline Sarcophagidae & 5,00 & $\mathrm{Nd}$ & $\mathrm{d}$ & $\mathrm{ac}$ & 10,54 & $\mathrm{D}$ & $\mathrm{ma}$ & $58,33 \mathrm{c}$ & 10,27 & $\mathrm{D}$ & $\mathrm{ma}$ & $25 \mathrm{a}$ \\
\hline Tephritidae & 39,00 & $\mathrm{D}$ & $\mathrm{ma}$ & $25 \mathrm{a}$ & 0,50 & nd & $\mathrm{d}$ & $25 \mathrm{a}$ & 0,02 & nd & $\mathrm{d}$ & $33,33 a$ \\
\hline Stratiomyidae & o & o & o & o & o & o & o & $\mathrm{o}$ & 8,00 & $\mathrm{D}$ & $\mathrm{ma}$ & $8,33 \mathrm{ac}$ \\
\hline Blattidae & 8,04 & $\mathrm{D}$ & $\mathrm{ma}$ & $66,67 \mathrm{c}$ & 1,80 & nd & $\mathrm{d}$ & $66,67 \mathrm{c}$ & 1,48 & nd & $\mathrm{d}$ & $66,67 \mathrm{c}$ \\
\hline Formicidae & 7,30 & $\mathrm{D}$ & $\mathrm{ma}$ & $66,67 \mathrm{c}$ & 3,34 & nd & $\mathrm{d}$ & $58,33 \mathrm{c}$ & 0,93 & nd & $\mathrm{d}$ & $58,33 \mathrm{c}$ \\
\hline Scolytidae & 14,00 & $\mathrm{D}$ & $\mathrm{ma}$ & $27 \mathrm{a}$ & o & o & o & o & o & o & o & o \\
\hline Gryllidae & 7,00 & nd & $\mathrm{d}$ & $66,67 \mathrm{c}$ & 0,50 & nd & $\mathrm{d}$ & $50 \mathrm{a}$ & 1,13 & nd & $\mathrm{d}$ & $58,33 \mathrm{c}$ \\
\hline Cicadellidae & 1,60 & nd & $\mathrm{d}$ & $33,33 \mathrm{a}$ & 2,13 & nd & $\mathrm{d}$ & $33,33 a$ & 0,58 & nd & $\mathrm{d}$ & $33,33 a$ \\
\hline Ichneumonidae & 2,30 & nd & $\mathrm{d}$ & $8,33 \mathrm{ac}$ & 0,80 & nd & $\mathrm{d}$ & $33,33 a$ & o & o & o & o \\
\hline Drosophilidae & o & o & o & o & 1,14 & nd & $\mathrm{d}$ & $58,33 \mathrm{c}$ & 0,18 & nd & $\mathrm{d}$ & $41,67 \mathrm{a}$ \\
\hline Chrysomelidae & 0,02 & nd & $\mathrm{d}$ & $25 \mathrm{a}$ & o & o & o & o & 0,55 & nd & $\mathrm{d}$ & $25 \mathrm{a}$ \\
\hline Curculionidae & 0,14 & nd & $\mathrm{d}$ & $66,67 \mathrm{c}$ & 0,10 & nd & d & $58,33 \mathrm{c}$ & 0,31 & nd & $\mathrm{d}$ & $50 \mathrm{a}$ \\
\hline Stenopelmatidae & o & o & o & o & o & o & o & o & 0,31 & nd & $\mathrm{d}$ & $33,33 a$ \\
\hline Forficulidae & 0,80 & nd & $\mathrm{d}$ & $25 \mathrm{a}$ & 0,10 & nd & d & $25 \mathrm{a}$ & $\mathrm{o}$ & o & o & o \\
\hline Sphecidae & o & o & o & o & o & o & o & o & 0,24 & nd & $\mathrm{d}$ & $8,33 \mathrm{ac}$ \\
\hline Scoliidae & $\mathrm{o}$ & o & o & o & o & o & o & o & 0,23 & nd & $\mathrm{d}$ & $8,33 \mathrm{ac}$ \\
\hline Carabidae & o & o & o & o & o & o & o & o & 0,14 & nd & $\mathrm{d}$ & $8,33 \mathrm{ac}$ \\
\hline Reduvidae & 0,02 & nd & $\mathrm{d}$ & $8,33 \mathrm{ac}$ & 0,01 & nd & $\mathrm{d}$ & $8,33 \mathrm{ac}$ & 0,03 & nd & $\mathrm{d}$ & $8,33 \mathrm{ac}$ \\
\hline Cercopidae & 0,42 & o & o & o & o & o & o & o & 0,05 & nd & $\mathrm{d}$ & $8,33 \mathrm{ac}$ \\
\hline
\end{tabular}

F = Frequência; $\mathrm{D}=$ Dominância: $\mathrm{d}=$ dominante; $\mathrm{nd}=$ não dominante; $\mathrm{A}=$ Abundância: $\mathrm{c}=$ comum; $\mathrm{d}=$ dispersa; ma = muito abundante; $\mathrm{C}$ = Constância $: \mathrm{c}=$ constante; $\mathrm{a}=$ acessória; $\mathrm{ac}=$ acidental; $\mathrm{o}=$ sem ocorrência. 
tos na estação chuvosa. Provavelmente, a maior frequência desses parasitoides deva-se a essa grande ocorrência do aracnídeo, promovendo assim, um equilíbrio populacional pelo inimigo natural. Já a presença das moscas califorídeas e sarcofagídeas deve-se a grande diversidade de animais silvestres mortos nessa vegetação, as quais são de suma importância para a ciclagem de energia no ecossistema do carrasco.

Os Formicidae, Blattidae, Drosophilidae e Curculionidae, apesar de apresentarem baixa frequência, foram constantes na vegetação do Carrasco, mas não dominaram e foram dispersos na vegetação; porém, desempenham importante papel na incorporação e ciclagem de matéria orgânica no solo. Como nessa vegetação existiam frutos em fermentação e árvores mortas, esses insetos têm um papel ecológico muito importante para esse ecossistema.

Já os Gryllidae, Cicadellidae, Ichneumonidae e Forficulidae ocorreram de forma acessória e, os Reduvidae, de forma acidental, mas todos não dominaram e foram dispersos na vegetação. As demais famílias estiveram ausentes (Tabela 4).

Na Mata Úmida, destacaram-se os Tephritidae, com uma frequência de 39\% (Tabela 4). Provavelmente, nessa vegetação e na estação chuvosa tenham surgido mais frutos hospedeiros silvestres do que nas outras vegetações, já que nessa família, encontram-se as chamadas moscas-das-frutas, insetos frugívoros, atacando um grande número de frutos hospedeiros, cultivados e silvestres. No entorno da FLONA, os produtores familiares cultivam frutas tropicais, dentre as quais se destaca a goiaba. De acordo com Azevedo et al. (2010), as espécies Anastrepha zenildae, A. obliqua, A. fraterculus, A. sororcula e Ceratitis capitata são comuns no município do Crato, atacando não somente essa fruta, mais outras da região do Cariri.

Em seguida a essa família, têm-se os Calliphoridae $(14,40 \%)$ e Scolytidae (14\%). A maior ocorrência dos escolitídeos deve-se a um maior número de árvores mortas nessa vegetação, já que algumas espécies contribuem na reciclagem de plantas mortas e a presença de espécies de Scolytidae está se tornando relativamente comum em plantações florestais e sua importância e abundância têm aumentado nos últimos anos (Flechtmann et al., 2001). Essa captura dos escolitídeos provavelmente ocorreu durante o voo para um novo hospedeiro, para constituir nova geração. De acordo com Flechtmann (1995), o estímulo para iniciar e permanecer em voo é governado pela temperatura, umidade relativa do ar e precipitação pluvial. Essas condições ótimas de sobrevivência e desenvolvimento dos insetos provavelmente foram encontradas na Mata Úmida durante a estação chuvosa. Essas famílias foram dominantes e muito abundantes, mas somente os Calliphoridae foram constantes na vegetação.
Os Blattidae e Formicidae também foram dominantes, muito abundantes e constantes nessa vegetação. Esses insetos têm importante papel na incorporação e ciclagem de matéria orgânica no solo, já que nessa vegetação existe grande concentração de serrapilheira no solo e a umidade da estação chuvosa favorece esse processo ecológico. Para faunas de formigas locais em florestas tropicais, até $50 \%$ podem estar associadas à serrapilheira (Delabie \& Fowler, 1995); aproximadamente 62\% de todas as espécies descritas no mundo habitam o solo e, ou, a serrapilheira (Wall \& Moore, 1999).

Os Gryllidae e Curculionidae foram constantes, enquanto os Cicadellidae, Chrysomelidae e Forficulidae ocorreram de forma acessória e os Sarcophagidae, Ichneumonidae e Reduvidae, acidentalmente, mas todos não dominaram e foram dispersos na vegetação. Os grilos, nessa época do ano e na Mata Úmida, não ocorreram com maior frequência como na estação seca porque, provavelmente, a ação das gotas de chuva reduziu a atividade desses insetos na vegetação, reduzindo a sua captura nas armadilhas de solo e, ou, bandejas. O mesmo pode ter ocorrido com os curculionídeos.

\section{CONCLUSÕES}

Os insetos da ordem Coleoptera são numerosos na estação seca, agindo como polinizadores de frutas (Nitidulidae), fitófagos (Curculionidae) e detritívoros (Scarabaeidae), além de decompositores de matéria orgânica na estação chuvosa.

Os Diptera são numerosos na estação chuvosa, atuando no sistema ecológico as moscas frugívoras (Drosophilidae e Tephritidae), decompositoras de carcaças de animais (Calliphoridae e Sarcophagidae) e de matéria orgânica (Stratiomyidae) e predadoras (Tachinidae).

Os Gryllidae predominam na Mata Úmida e os Formicidae no Carrasco e Cerrado da estação seca.

Os Calliphoridae predominam no Cerrado, os Tachinidae no Carrasco e os Tephritidae na Mata Úmida, na estação chuvosa.

Apesar de alguns táxons não predominarem, cada um desempenha importante papel ecológico sobre as vegetações, nas estações do ano.

\section{AGRADECIMENTOS}

À Chefe do IBAMA, Verônica Maria Figueiredo, pela disponibilização das áreas para a realização das coletas na Floresta Nacional do Araripe. Ao analista ambiental Carlos Leal Filho, pelo acompanhamento da pesquisa nessas áreas, e ao voluntário Sr. Chico, pelo auxílio na instalação das armadilhas e na coleta dos insetos no campo. 


\section{REFERÊNCIAS}

Azevedo FR, Guimarães JA, Simplício AAF \& Santos HR (2010) Análise faunística e flutuação populacional de moscas-das-frutas (Diptera:Tephritidae) em pomares comerciais de goiaba na região do Cariri cearense. Arquivos do Instituto Biológico, 77:33-41.

Bruyn LAL (1999) Ants as bioindicators of soil function in rural environments. Agriculture, Ecosystems and Environment, 74:425-441.

Buzzi ZJ (2008) Entomologia didática. 4 ${ }^{\mathrm{a}}$ ed. Curitiba, Editora UFPR. 348p

Catts EP \& Goff ML (1992) Forensic entomology in criminal investigations. Annual Review of Entomology, 37:253-272.

Chung AYC, Eggleton P, Speight MR, Hammond PM \& Chey VK (2000) The diversity of beetle assemblages in different habitat types in Sabah, Malaysia. Bulletin of Entomological Research, 90:475-496.

Coelho LBN \& Silva ER (2003) Flutuação populacional de Agallia incongrua Oman, 1938 (Hemiptera: Cicadellidae) em Viçosa, Minas Gerais, Brasil. Biota Neotropica, 3:1-8.

Delabie JHC \& Fowler HG (1995) Soil and litter cryptic ant assemblages of Bahian cocoa plantations. Pedobiologia, 39:423-433.

Flechtmann CAH (1995) Scolytidae em reflorestamentos com pinheiros tropicais. Piracicaba, IPEF. 201p.

Flechtmann CAH, Ottati ALT \& Berisford CW (2001) Ambrosia and bark beetles (Scolytidae: Coleoptera) in pine and eucalypt stands in southern Brazil. Forest Ecology and Management, 142:183-191.

Halter G \& Arellano L (2002) Response of dung beetle diversity to humanin duced changes in a tropical landscape. Biotropica, 34:144-154.

Kimberling DN, Karr JR \& Fore LS (2001) Measuring human disturbance using terrestrial invertebrates in the shrub-steppe of eastern Washington (USA). Ecological Indicators, 19:63-81.

Küchmeister H, Webber AC, Silberbauer-Gottsberger I \& Gottsberger G (1998) A polinização e sua relação com a termogênese em espécies de Arecaceae e Annonaceae da Amazônia Central. Acta Amazônica, 28:217-245.

Lima MF, Lima FAM \& Teixeira MMS (1984) Mapeamento e demarcação definitiva da Floresta Nacional do Araripe, Ceará, Brasil. Ciência Agronômica, 15:59-69.

Lopes BGC (2008) Levantamento da entomofauna bioindicadora da qualidade ambiental em diferentes áreas do alto JequitinhonhaMinas Gerais. Monografia de graduação, Escola Agrotécnica Federal de Inconfidentes, Inconfidentes, 47p.

Milhomem MS, Mello FZV \& Diniz IR (2003) Técnicas de coleta de besouros copronecrófagos no Cerrado. Pesquisa Agropecuária Brasileira, 38:1249-1256.

Nadel H \& Peña JE (1994) Identity, behavior, and efficacy of nitidulid beetles (Coleoptera: Nitidulidae) pollinating commercial Annona species in Florida. Environmental Entomology, 23:878-886.

Oberprieler RG, Marvaldi AE \& Anderson RS (2007) Weevils, weevils, weevils every where. Zootaxa, 1668:1-766.

Paschoalini EL, Fontonelle JC, Castro FS, Carvalho CS, Costa ILL, Almeida JC, Sillotto H \& Martins RP (2004) Variação anual e estacional no número de indivíduos de famílias de Diptera (Brachycera e Cychlorrapha) usando Malaise. In: Congresso Brasileiro de Zoologia Brasília, Brasília. Anais, UNB. p.97.

Pujol-Luz JR, Xerez R \& Viana GG (2004) Descrição do pupário de Raphiocera armata (wiedemann) (Diptera, Stratiomyidae) da Ilha da Marambaia, Rio de Janeiro, Brasil. Revista Brasileira de Zoologia, 21:995-999.
Ricklefs RE (2001) A economia da natureza. 5 ${ }^{\mathrm{a}}$ ed. Rio de Janeiro, Guanabara Koogan. 470p.

Rozkosny R (1982) A biosystematic study of the European Stratiomyidae (Diptera). Séries Entomológica, 21:1-401.

Sakagami SF \& Laroca S (1971) Relative abundance, phenology and flower visits of apid bees in Eastern Paraná, South Brazil (Hym., Apidae). Kontyü, 39:213-230.

Silveira Neto S, Nakano O, Bardin D \& Vila Nova NA (1976) Manual de ecologia dos insetos. São Paulo, Agronômica Ceres. 420 p.

Soares IMF, Gomes DS \& Santos AA (2001) Influência da composição florística na diversidade de formigas (Hymenoptera: Formicidae) na Serra da Jibóia-BA. In: $15^{\circ}$ Encontro de Mirmecologia, Londrina, Resumos, IAPAR, p.331-332.

Schorn LA (2000) Fatores que afetam a produção de sementes (Curso de manejo e conservação de sementes de espécies arbóreas da Mata Atlântica - Região Sul), 1. Blumenau. 47p.

Shorrocks B (1974) Niche parameters in domestic species of Drosophila. Journal of Natural History, 8:215-222.

Smith KGV (1986) A manual of forensic entomology. Ithaca, USA, Cornell University Press. 205p.

Sperber CF (1999) Por que há mais espécies de grilo (Orthoptera: Grylloidea) em fragmentos florestais maiores? Dissertação de Mestrado. Universidade Estadual Paulista, Rio Claro, 295p.

Sperber CF, Soares LGS \& Pereira MR (2007) Litter disturbance and trap spatial positioning affects number of captured individuals and genera of crickets (Orthoptera: Grylloidea). Journal of Orthoptera Research, 16:1-7.

Thomanzini MJ \& Thomanzini APBW (2000) A fragmentação florestal e a diversidade de insetos nas florestas tropicais úmidas. Rio Branco, EMBRAPA Acre, 21p. (Circular Técnica, 57).

Thomanzini MJ \& Thomanzini APBW (2002) Levantamento de insetos e análise entomofaunística em floresta, capoeira e pastagem no Sudeste Acreano. Rio Branco, EMBRAPA Acre. 41p. (Circular Técnica, 35).

Tidon R (2006) Relationships between drosophilids (Diptera, Drosophilidae) and the environment in two contrasting tropical vegetations. Biological Journal of the Linnean Society, 87:233-247.

Triplehorn CA \& Johnson NF (2011) Estudo dos insetos. São Paulo, Cengage Learning. 809p.

Vaz VVA, Dummel K, Nunes MR, Gantes ML, Oliveira EA, Carrasco DS \& Zardo CML (2009) Comparação de Cicadellidae (Hemiptera; Auchenorrhyncha) em duas ilhas com diferentes composições florísticas, situadas no estuário da laguna lagoa dos patos, RS, Brasil. In: $9^{\circ}$ Congresso de Ecologia do Brasil, São Lourenço - Minas Gerais, Anais, CD-ROM.

Wall DH \& Moore JC (1999) Interactions underground. BioScience, 49:109-107.

Whittaker RH (1977) Evolution of species diversity in land communities. Evolutionary Biology, 10:1-67.

Wink C, Guedes JVC, Fagundes CK \& Rovedder AP (2005) Insetos edáficos como indicadores da qualidade ambiental. Revista de Ciências Agroveterinárias, 4:60-71.

Yamamoto LF (2001) Florística e síndromes de polinização e dispersão em um fragmento de floresta estacional semidecídua montana, Município de Pedreira, Estado de São Paulo. Dissertação de Mestrado. Universidade de Campinas, Campinas, 83p.

Zidko A (2002) Coleópteros (Insecta) associados às estruturas reprodutivas de espécies florestais arbóreas nativas no Estado de São Paulo. Dissertação de Mestrado, Escola Superior de Agricultura Luiz de Queiroz, Piracicaba, 35p. 